\title{
Stock Trading System Based on Formalized Technical Analysis and Ranking Technique
}

\author{
Saulius Masteika and Rimvydas Simutis \\ Faculty of Humanities, Vilnius University, \\ Muitines 8, 44280 Kaunas, Lithuania \\ saulius.masteika@vukhf.1t, rimvydas.simutis@vukhf.lt
}

\begin{abstract}
The contribution of this paper lies in a novel application of formalized technical analysis and ranking technique by development of efficient stock trading system. The proposed system is implemented using two steps: on the first step system analyses historical data from large number of stocks and defines a quality function of each stock to specific technical trade pattern; on the second step system grades all the stocks according to the value of the defined quality function and makes suggestions to include the highest ranked stocks into the traders' portfolio. These stocks are being hold for fixed time interval in traders' portfolio, then sold and replaced with the new stocks that got the highest rank. The proposed trading system was tested using historical data records from the USA stock market (1991-2003). The trading system had given significantly higher returns when compared to the benchmark.
\end{abstract}

\section{Introduction}

The continuing progress of computing methods and information technologies makes significant influence on financial markets. An effective information processing is more and more important in order to succeed in the stock markets. The strategists of the investments funds use historical data and real-time information to forecast the trends of stocks' price changes and also apply these results to the decision making and formation of investment portfolios. However wide known Efficient Market Hypothesis (EMH) states that prices of stocks are just a reflection of all known information about the company. This means that having the information, no prediction of future price changes can be made. EMH also states that new information immediately forms new prices of companies' shares. If we agree with this hypothesis, we have to realize that analysis of historical information is not important for an investor as this information is already reflected in the stock price. People are not able to forecast the flow of new, important information and this information appears randomly. Therefore according to the Efficient Market Hypothesis it is impossible to get better returns in the stock market than the market benchmarks (e.g. S\&P500 index) for a longer time period using the available historical information about the market. There are numerous well known research papers [1], [2], that partly confirm this hypothesis. However some published reports show [3], [4], that the efficient market hypothesis is far from the correct one. There are some papers claiming that the application of technical 
analysis or nonlinear models, such as neural networks and genetic algorithms, can supply information for intelligent decision support systems, and traders using these techniques are able to beat the market for a long period of time [5], [6], [7], [8]. In this paper we reinforce these statements. The contribution of this paper lies in novel application and combination of formalized technical analysis and ranking technique for development of efficient stock trading system. The proposed system is quite simple in implementation and could attract an attention of individual stock traders and modest mutual funds. The proposed stock trading system was tested using historical data from USA stock market. The obtained results clearly contradict the statements of Efficient Market Hypothesis. The trading system based on the proposed approach has given significantly higher returns relative to the benchmark (S\&P500) for a long period of time. In order to use the system in real markets it is necessary to solve problems with the risk management of trader's portfolio and to carry out additional experimental tests with the stock markets of different countries and different time periods. The paper is organized as follows: Section 2 provides the theoretical basis of the proposed system; Sections 3 is devoted for experimental investigations and evaluation of the proposed system. The main conclusions of the work are presented in Section 4.

\section{Development of the Stock Trading System}

The proposed stock trading system is based on formalized technical analysis and is suited for medium and short term stock traders. Over the years numerous technical indicators and patterns have been developed to describe stock performance, as well as to predict future price movements. Technical analysts and traders believe that certain stock chart patterns and shapes (e.g. moving average crosses, head and shoulder formation, range breakout, triple top/bottom formation, cup-with-a-handle formation) are signals for profitable trading opportunities [9], [10]. Many professional traders claim that they consistently make trading profits by following those signals. Short term stock traders analyze historical stock data records (price and volume) for 5-7 trading days and make decisions for buying stocks based on typical trading patterns. Normally they hold stocks for $2 \div 5$ days. In this paper we introduce a new technical pattern, called "precursor of reverse" pattern. This pattern can be characterized as follows: after a long price decline (5-6 days) with strong trading volume, the stock price and trading volume stabilize and price prepares for rebound. The Figure 1 illustrates a typical example of the "precursor of reverse" pattern. Our extensive and rigorous historical tests have shown that this pattern has a good rebound potential. The stock trading strategy is to buy the stock as the price and volumes stabilize and hold it for 2-5 days.

\subsection{Formalization of the Trading System}

Classical technical analysis compares stock's price and volume history to typical chart patterns (in our case "precursor of reverse" pattern) and predicts future price behavior based on the degree of match. This technique can be formalized through trading rule of the following form: If the technical pattern $X$ is identified in the previous $N$ trading days, then buy the stock and sell it on the H-th day after purchasing. The difficult part in application of this technique is reliability of identification of the technical pattern $X$. 

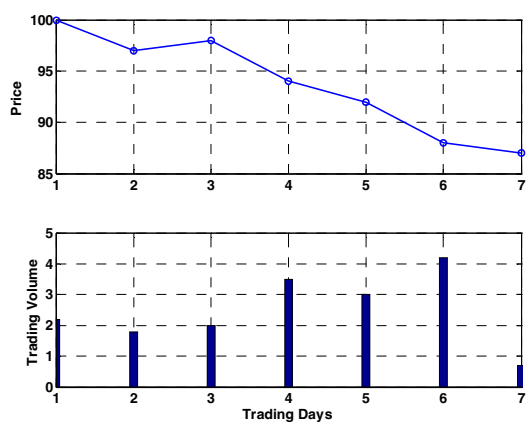

Fig. 1. A typical "precursor of reverse" pattern

The new technique that we propose here is based on the formalization of "precursor of reverse" pattern using normalized historical stock prices and trading volume data. The idea of this technique is to analyze $\mathrm{N}$-days moving window for a big pool of stocks with normalized closing price and volume records and identify the technical pattern when it appears. For formalization of the "precursor of reverse" pattern we use three variables: normalized stock price $P_{n N}$, normalized trading volume $V_{n N}$ at the last day of observation and normalized price change during the last day $D_{n N}$. During the $N$-days moving window the normalized stock prices $P_{n i}$, normalized trading volume $V_{n i}$ and normalized price changes $D_{n i}$ are obtained using the following equations :

$$
P_{n i}=\frac{P_{i}}{P_{1}} \cdot 100, \quad V_{n i}=\frac{V_{i}}{\frac{\sum_{i=1}^{N} V_{i}}{N}}, D_{n i}=P_{n(i-1)}-P_{n i} \quad i=1 \ldots N,
$$

where $P_{i}$ and $V_{i}$ are stocks' closing prices and trading volumes on $i$-day. This equation is estimated every day for all stocks in pool. We assume that a "precursor of reverse" pattern is formed when the stock price drops approximately $10 \%$ during the seven trading days, the last day's price drops about $2 \%$ and the trading volume on last day of observation begins to decrease. In traditional technical analysis the quality of the formed pattern is evaluated using various graphical methods. Testing and application of such methods are complicated because different traders often evaluate the same trading pattern in different ways. In this paper we propose a formal equation for evaluation of quality of trading pattern $Q_{p}$. This equation is derived based on extensive interviews with experienced stock trading experts and has a following form:

$$
Q_{p}=\frac{\left(P_{n 1}-P_{n N}\right)}{K_{N}+\left(P_{n 1}-P_{n N}\right)} \cdot \frac{D_{n N}}{K_{D}+D_{n N}} \cdot \frac{K_{V}}{K_{V}+V_{n N}} .
$$

As it can be recognized from the structure of the equation, the quality of the "precursor of reverse" pattern' $Q_{p}$ is more precise when stock's price drops significantly during the observed time interval and stock's price also decrease at the last day of observation, but with decreasing trading volume. The values of parameters $K_{N}, K_{D}, K_{V}$ in Equation 2 were defined using the recommendation of trading experts and are given in experimental part of this work. Having stock data records for defined moving time interval now it is straightforward to estimate the three variables of proposed 
pattern for every trading day and define the quality of the pattern, $Q_{P}$. This information is the backbone of the proposed trading system.

\subsection{Ranking Technique}

Experimental investigations of the "precursor of reverse" patterns had shown that high quality patterns are very rare. Still further, approximately only the $70 \%$ of the high quality patterns are profitable. Therefore we need to scan a lot of stocks to find potentially profitable trading opportunities. The proposed algorithm for stocks ranking consists of the following steps:

- $\quad$ in the first step a group of companies, whose trading patterns will be observed is selected,

- then the trading patterns - "precursor of reverse" are identified, using the moving window data,

- $\quad$ the quality of each trading pattern is estimated (Equation 2),

- all trading patterns are ranked following the highest pattern quality and the highest rank stocks are suggested to the trader.

\subsection{Evaluation of the Trading Strategy}

A portfolio of the stock trader is formed of stocks having highest rank of trading pattern. Number of stocks held in portfolio will be discussed in experimental part of this work. The efficiency of the proposed system was evaluated using three parameters: a total return on trading $R_{T}$, a total capital at the end of trading period $C_{T}$ and Sharpe Ratio of the trader's portfolio $S$. The total return $R_{T}$ was estimated using equation

$$
R_{T}=\sum_{i=1}^{T} r_{i}, \quad r_{i}=\frac{P_{i}-P_{i-1}}{P_{i-1}} \cdot 100-T_{C},
$$

where $T$ is the number of trading transactions, $P_{i}$ is stock price, $T_{C}$ is transaction costs and $r_{i}$ is simple return of $i$-th transaction. Total capital at the end of the trading period was estimated using trader's start capital and every day returns. The Sharpe Ratio is a well known measure of the risk-adjusted return of an investment [11]. Sharpe Ratio of the trader's portfolio was estimated using equation

$$
S(t)=\frac{R_{D}(t) \cdot 250-R_{a}}{\sigma_{D}(t) \cdot \sqrt{250}},
$$

where $R_{D}$ and $\sigma_{D}$ - average daily returns and standard deviation of the trader's portfolio over moving 250 days, $R_{a}$ - annualized return on "risk-free" investment (we assumed it to be $3 \%$ ). Mutual funds which achieve a Sharpe Ratio of over 1.0 are qualified as good. A Sharpe Ratio of a fund of over 2.0 is considered very good and Sharpe Ratios above 3.0 are outstanding.

\section{Experimental Investigation}

The efficiency of the proposed stock trading system was tested using historical stock market data. The data set that has been used in this study represents daily stock clos- 
ing prices and trading volumes, and comes from 366 stocks on the USA stock market from October 1, 1991 till October 1, 2003. These stocks represent all the SP500 index stocks, included in the index for the whole analyzed time period.

\subsection{Experimental Set-Up}

We have used 7-days moving window technique and normalization technique (Eq.1) to identify the "precursor of reverse" patterns in data records. The quality of the patterns, $Q_{P}$ was evaluated using Equation 2. The values of parameters here were chosen empirically using extensive interviews with stock trading experts. The values of parameters are as follows: $K_{N}=10.0, K_{D}=2.0, K_{V}=3.5$. Because the values of these parameters are crucial for the trading system more investigation must be done in the future for the optimization and adaptation of them. All trading patterns were ranked; the stocks with highest ranks were included in trader's portfolio and held for a defined time interval. The performance of the trading system was evaluated using proposed parameters, $R_{T}, C_{T}$ and $S$. The detailed functioning of the proposed trading system can be described as follows:

- The stocks' prices and trading volumes were normalized and analyzed considering every trading day starting from 1991.10.01 to 2003.10.01;

- Based on 7-days moving window data (normalized price and volume) the "precursor of reverse" patterns were identified and quality of these patterns, $Q_{P}$, for every stock were estimated;

- $\quad$ Ranking algorithm for $Q_{P}$ was applied and stocks (1-15 stocks) with the best ranks were bought and sold after defined time interval (1-5 days).

In the beginning of the testing period the start capital for trader was 1000 US\$. Equal parts of this capital were invested in all selected stocks. The transaction costs of $0.5 \%$ were withdrawn from the returns per contract. This amount of transaction costs is typical for e-brokers companies (e.g. Interactive Brokers, USA) and reflects realistic losses that are unavoidable when changing the stocks in trader's portfolio. All income got during the stocks' trading actions were again completely reinvested and the changing of the total capital was estimated during the investment period. The structure of the proposed virtual stock trading system is presented in Figure 2. It is necessary to point out that the proposed scheme gives the possibility to apply the proposed system in real stock trading process. In this case the highest ranked stocks calculated on 7-days moving window data can be bought using market orders at the last moment before the markets are closed.

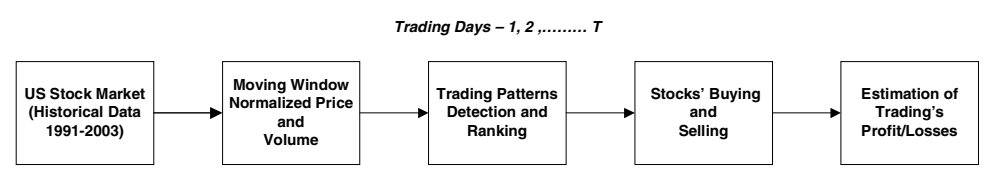

Fig. 2. The structure of the proposed stock trading system 


\subsection{Experimental Results}

The total returns of proposed trading system are presented in Figure 3. The best results are achieved when trader buys the five best ranked stocks every day and hold them for two days. In this case the proposed trading system outperforms the benchmark significantly and the total return at the end of the trading period is $742 \%$.The total return for the SP500 index during the same period is $112 \%$, and total return when investing (buy and hold) in all analyzed stocks (equal part of capital in each stock) is $265 \%$. Trading a smaller number of best ranked stocks (e.g. 1 to 4 ) gives even better results, but this leads to a big fluctuation in portfolio performance, therefore is not interesting for real trading process and will not be discussed here.

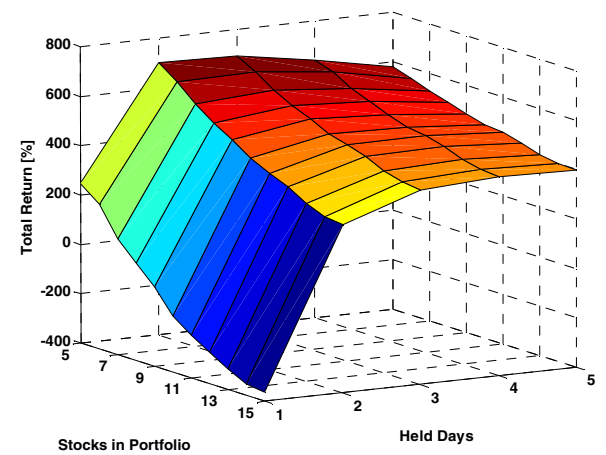

Fig. 3. The total returns depends on number of obtained stocks and how long the obtained stocks are held in trader's portfolio

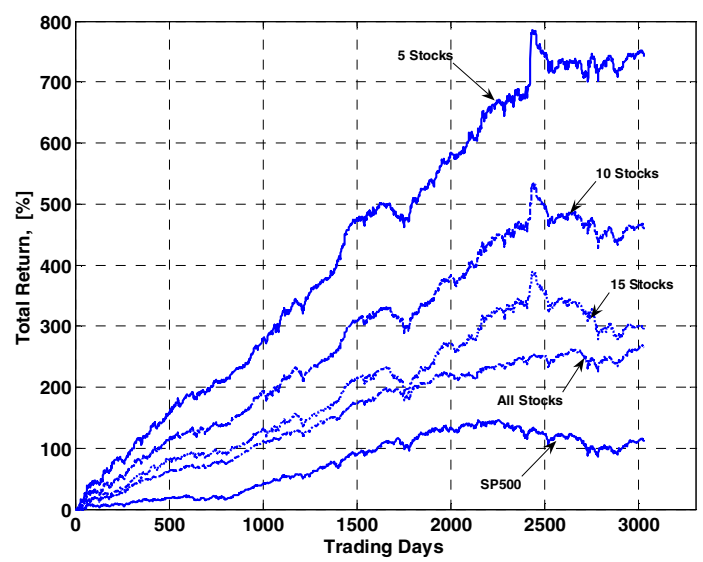

Fig. 4. The dynamic of total returns during the whole trading period for various portfolios

Figure 4 presents how the total returns of proposed trading system change during the analyzed time interval (obtained stocks are held for two days). As one can see 


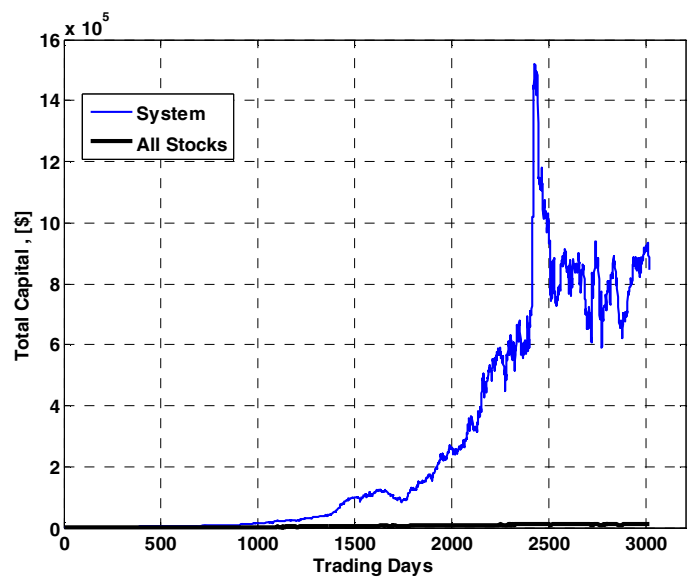

Fig. 5. The dynamic of total capital when using proposed trading strategy and conservative investment (all stocks are held for whole trading period)

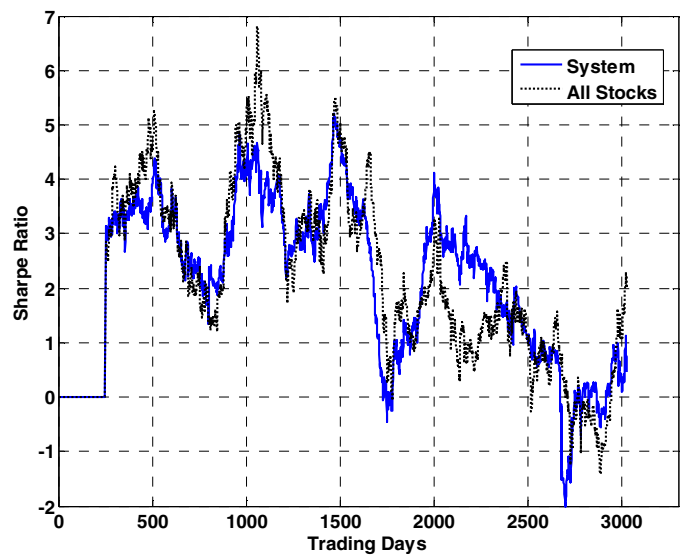

Fig. 6. Sharpe Ratio estimated for proposed trading system and for conservative investments

from the graph, the fluctuation in total returns decrease when more different stocks are included in the trader's portfolio. On the other side, the including of more different stocks in the trader's portfolio leads to the declining in total returns. Therefore the trader must always make well-balanced decision about possible portfolio fluctuations and returns, and choose the adequate number of traded stocks. Figure 5 compares the dynamic of trader's capital when trading the highest ranked five stocks and when using conservative investment strategy (start capital is invested in all analyzed stocks). The trader's capital using the proposed trading system increases dramatically from $1000 \$$ to $848610 \$$. At the same time conservative investment gives the end capital equal 13060 \$. Figure 6 illustrates the Sharpe Ratio estimation for both: proposed trading system and conservative investments. As it can be recognized from the 
graph the Sharpe Ratio of the proposed trading system is relative high and is similar to the Ratio obtained for conservative investments. Nonetheless, the Sharpe Ratio decreases significantly for some periods of time and these periods can be very hard challenges for individual traders or mutual funds.

\section{Conclusions}

Preliminary experimental tests have shown that the proposed stock trading system gives very promising results and can be attractive for individual traders and modest mutual funds. Despite of these results there are two problems that will arise while using the proposed system in real stock markets: a) trading results are very sensitive to the costs of transactions: when transaction costs are over $0.8 \%$ the results of trading system's become worse than investing in benchmark; b) proposed trading system is related with high risks and the traders must be prepared for temporal decreasing of total capital even to $50 \%$ (Fig. 5). Also more extensive tests with the system must be done (different markets, various time periods, tax regulations) to evaluate the domain of possible applications of the proposed trading system more precisely.

\section{References}

1. White, H. Economic prediction using neural networks: The case of IBM daily stock returns. In: IEEE International Conference on Neural Networks. San Diego, 1988, pp. 451459.

2. Lowe, A.R.Webb. Time series prediction by adaptive networks: a dynamical systems perspective. In Artificial Neural Networks: Forecasting Time Series (eds. V. Rao Vemuri and Robert D. Rogers). IEEE Computer Society Press, 1994, pp. 12-19. D.

3. F. Fama. Efficient capital markets, Journal of Finance, 46(5),1991, 1575-1617.

4. D.J. Baestaens, W.M. van den Bergh, H. Vaudrey. Market inefficiencies, technical trading and neural networks. In: Dunis, C. (ed.): Forecasting Financial Markets, Financial Economics and Quantitative Analysis, Chichester, John Wiley \& Sons, 1996, pp. 245-260.

5. A.Refenes. Neural Networks in the Capital Markets, Wiley, Chichester, 1995.

6. W. Leigh, R. Purvis, J.M. Ragusa. Forecasting the NYSE composite index with technical analysis, pattern recognizer, neural network, and genetic algorithm: a case study in romantic decision support. Decision Support Systems, 32, 2002, 361-377.

7. H. Hong. J. Stein. A unified theory of underreaction, momentum trading, and overreaction in asset markets, The Journal of Finance LIV (6), 1999, 2143-2184.

8. H. Hong, T. Lim, J. Stein. Bad news travels slowly: size, analyst coverage, and the profitability of momentum strategies. The Journal of Finance LV (1), 2000, 265-295.

9. S.B. Achelis. Technical Analysis from A to Z, 2nd Editon, McGraw-Hill Professional, 2000.

10. R. Martinelli, B. Hyman. Cup-with-handle and the computerized approach. Technical Anglysis of Stocks and Commodities, 16(10), 1999, 63-66.

11. Sharpe W. F. Asset allocation: Management Style and Performance Measurement," Journal of Portfolio Management, Winter 1992, pp. 7-19. 